\title{
Trijugin-type limonoids from the leaves of Cipadessa cinerascens
}

Ying-Tong Di, ${ }^{\dagger, \dagger}$ Hong-Ping He, ${ }^{\dagger}$ Hai-Yang Liu, ${ }^{\dagger}$ Ping Yi, ${ }^{\dagger}$ Zhen Zhang, ${ }^{\S}$ Yan-Li

Ren, ${ }^{\perp}$ Jun-Song Wang, ${ }^{\dagger}$ Qian-Yun Sun, $\|$ Fu-Mei Yang, $\|$ Xin Fang, ${ }^{\dagger}$ Shun-Lin Li, ${ }^{\dagger}$

Hua-Jie Zhu, ${ }^{\dagger}$ and Xiao-Jiang Hao ${ }^{* \dagger}$

State Key Laboratory of Phytochemistry and Plant Resources in West China,

Kunming Institute of Botany, Chinese Academy of Sciences, Kunming 650204,

People's Republic of China, Graduate School of Chinese Academy of Sciences,

Beijing 100039, People's Republic of China, College of Pharmaceutical Sciences,

Dali University, Dali 677100, People's Republic of China, College of Living Creature

Science and Technology, Hunan Agricultural University, Changsha 410128, Hunan,

People's Republic of China, and Key Laboratory of Chemistry for Natural Products

of Guizhou Province and Chinese Academy of Sciences, Guiyang 550002, People's

Republic of China

${ }^{*}$ Corresponding author. Tel.: +86-871-5223263. Fax: +86-871-5219684. E-mail:

haoxj@mail.kib.ac.cn.

${ }^{\dagger}$ Kunming Institute of Botany.

$¥$ Graduate School of Chinese Academy of Sciences.

${ }^{\S}$ College of Phamaceutical Sciences, Dali University.

${ }^{\perp}$ College of Living Creature Science and Technology, Hunan Agricultural University.

\| Key Laboratory of Chemistry for Natural Products of Guizhou Province and Chinese Academy of Sciences. 


\section{Supporting Information}

Figure S1. $\quad{ }^{1} \mathrm{H}$ NMR spectrum (Bruker AM-500, $500 \mathrm{MHz}, \mathrm{CDCl}_{3}$ ) of Cipatrijugin A (1)......3

Figure S2. $\quad{ }^{13} \mathrm{C}$ NMR spectrum (Bruker AM-400, $100 \mathrm{MHz}, \mathrm{CDCl}_{3}$ ) of Cipatrijugin A (1).....3

Figure S3. $\quad{ }^{1} \mathrm{H}-{ }^{1} \mathrm{H}$ COSY spectrum (Bruker AM-500, $500 \mathrm{MHz}, \mathrm{CDCl}_{3}$ ) of Cipatrijugin A (1)..4

Figure S4. HMQC spectrum (Bruker AM-500, $500 \mathrm{MHz}, \mathrm{CDCl}_{3}$ ) of Cipatrijugin A (1).......4

Figure S5. $\mathrm{HMBC}$ spectrum (Bruker AM-500, $500 \mathrm{MHz}, \mathrm{CDCl}_{3}$ ) of Cipatrijugin A (1)........5

Figure S6. ROESY spectrum (Bruker AM-500, $500 \mathrm{MHz}, \mathrm{CDCl}_{3}$ ) of Cipatrijugin A (1)........5

Figure S7. $\quad{ }^{1} \mathrm{H}$ NMR spectrum (Bruker AM-500, $500 \mathrm{MHz}, \mathrm{CDCl}_{3}$ ) of Cipatrijugin B (2)......6

Figure S8. $\quad{ }^{13} \mathrm{C}$ NMR spectrum (Bruker AM-400, $100 \mathrm{MHz}, \mathrm{CDCl}_{3}$ ) of Cipatrijugin B (2)....6

Figure S9. HMQC spectrum (Bruker AM-500, $500 \mathrm{MHz}, \mathrm{CDCl}_{3}$ ) of Cipatrijugin B (2).......7

Figure S10. $\mathrm{HMBC}$ spectrum (Bruker AM-500, $500 \mathrm{MHz}, \mathrm{CDCl}_{3}$ ) of Cipatrijugin B (2).......8

Figure S11. ${ }^{1} \mathrm{H}$ NMR spectrum (Bruker AM-500, $500 \mathrm{MHz}, \mathrm{CDCl}_{3}: \mathrm{CD}_{3} \mathrm{OD}(9: 1)$ ) of Cipatrijugin C (3) .8

Figure S12. ${ }^{13} \mathrm{C}$ NMR spectrum (Bruker AM-400, $100 \mathrm{MHz}, \mathrm{CDCl}_{3}: \mathrm{CD}_{3} \mathrm{OD}(9: 1)$ ) of Cipatrijugin C (3) .9

Figure S13. HMQC spectrum (Bruker AM-500, $500 \mathrm{MHz}, \mathrm{CDCl}_{3}: \mathrm{CD}_{3} \mathrm{OD}(9: 1)$ ) of Cipatrijugin $\mathrm{C}(3)$ .9

Figure S14. HMBC spectrum (Bruker AM-500, $500 \mathrm{MHz}, \mathrm{CDCl}_{3}: \mathrm{CD}_{3} \mathrm{OD}(9: 1)$ ) of Cipatrijugin C (3). 10

Figure S15. ${ }^{1} \mathrm{H}$ NMR spectrum (Bruker AM-500, $500 \mathrm{MHz}, \mathrm{CDCl}_{3}$ ) of Cipatrijugin D (4).....11

Figure S16. ${ }^{13} \mathrm{C}$ NMR spectrum (Bruker AM-400, $100 \mathrm{MHz}, \mathrm{CDCl}_{3}$ ) of Cipatrijugin D (4).....11 Figure S17. $\quad{ }^{1} \mathrm{H}-{ }^{1} \mathrm{H}$ COSY spectrum (Bruker AM-500, $500 \mathrm{MHz}, \mathrm{CDCl}_{3}$ ) of Cipatrijugin D 


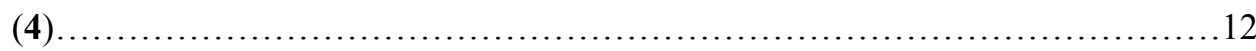

Figure S18. HMQC spectrum (Bruker AM-500, $500 \mathrm{MHz}, \mathrm{CDCl}_{3}$ ) of Cipatrijugin D (4).....12

Figure S19. HMBC spectrum (Bruker AM-500, $500 \mathrm{MHz}, \mathrm{CDCl}_{3}$ ) of Cipatrijugin D (4)......13

Figure S20. ROESY spectrum (Bruker AM-500, $500 \mathrm{MHz}, \mathrm{CDCl}_{3}$ ) of Cipatrijugin D (4).....13

Figure S21. Energy and Standard Orientation of Cipatrijugin A (1) .......................

Figure S22. Energy and Standard Orientation of Cipatrijugin D (4) E..................... 17 
Figure S1. ${ }^{1}$ H NMR spectrum of Cipatrijugin A (1).

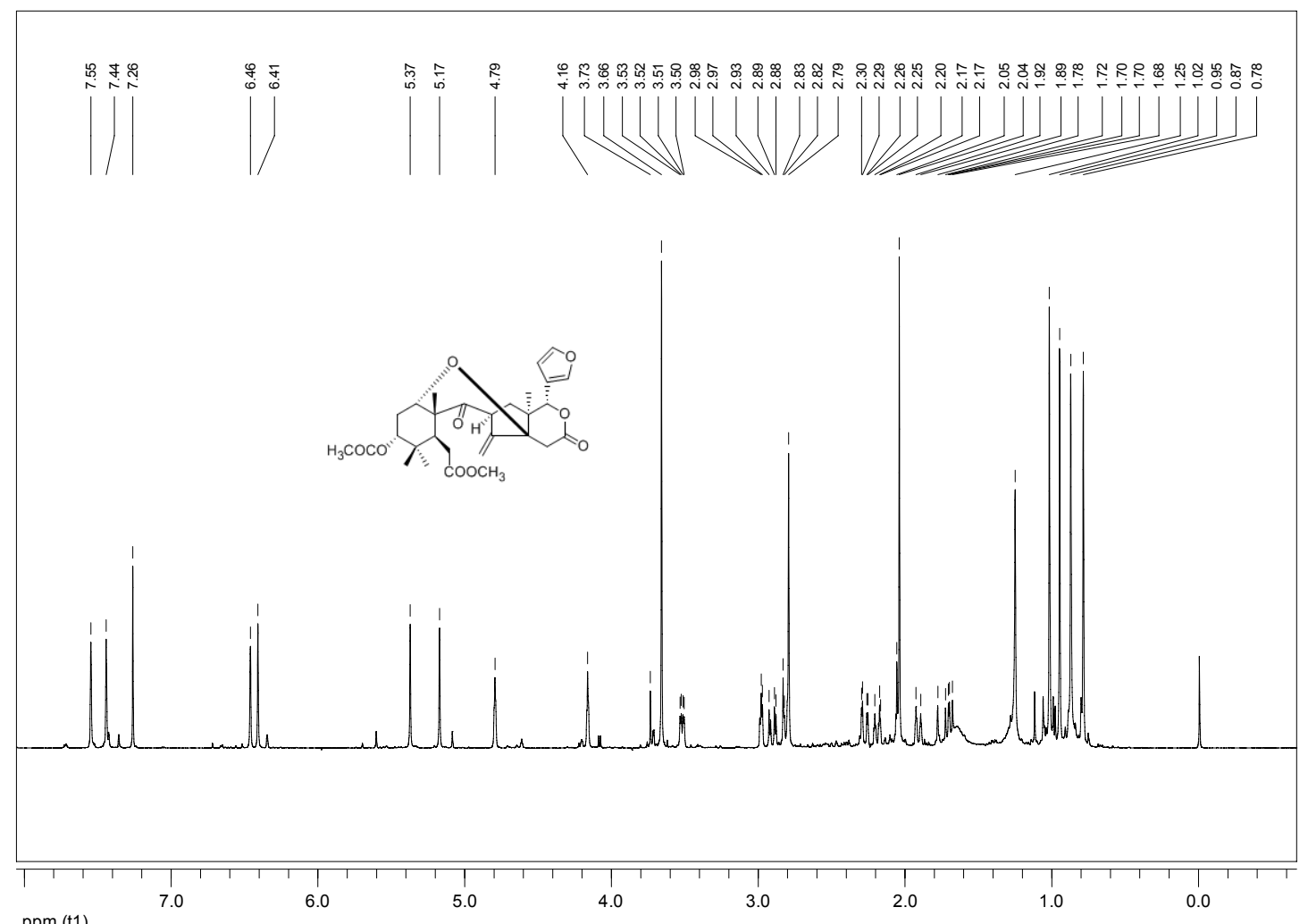

Figure S2. $\quad{ }^{13} \mathrm{C}$ NMR spectrum of Cipatrijugin A (1)

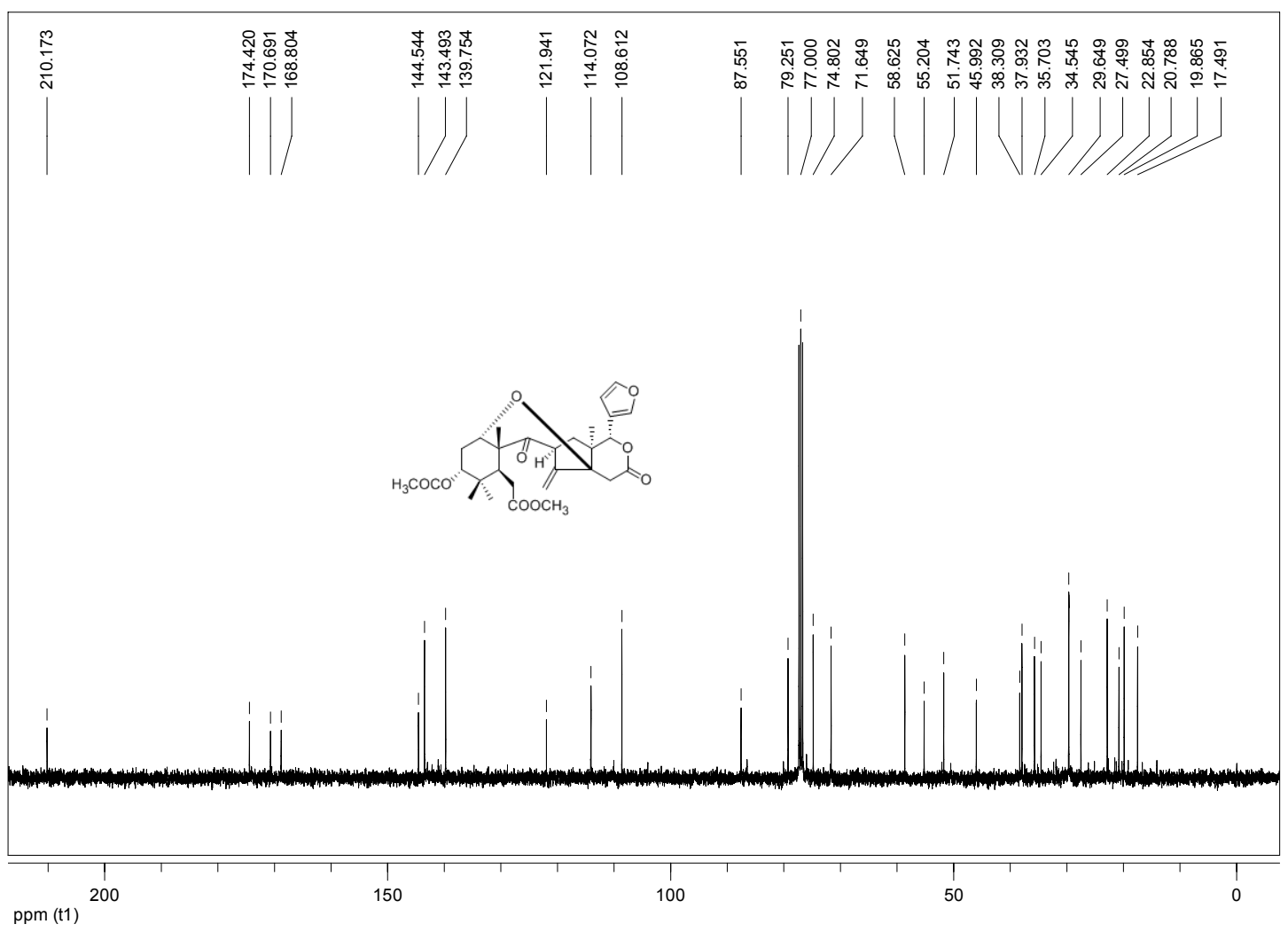


Figure S3. ${ }^{1} \mathrm{H}-{ }^{1} \mathrm{H}$ COSY spectrum of Cipatrijugin A (1)

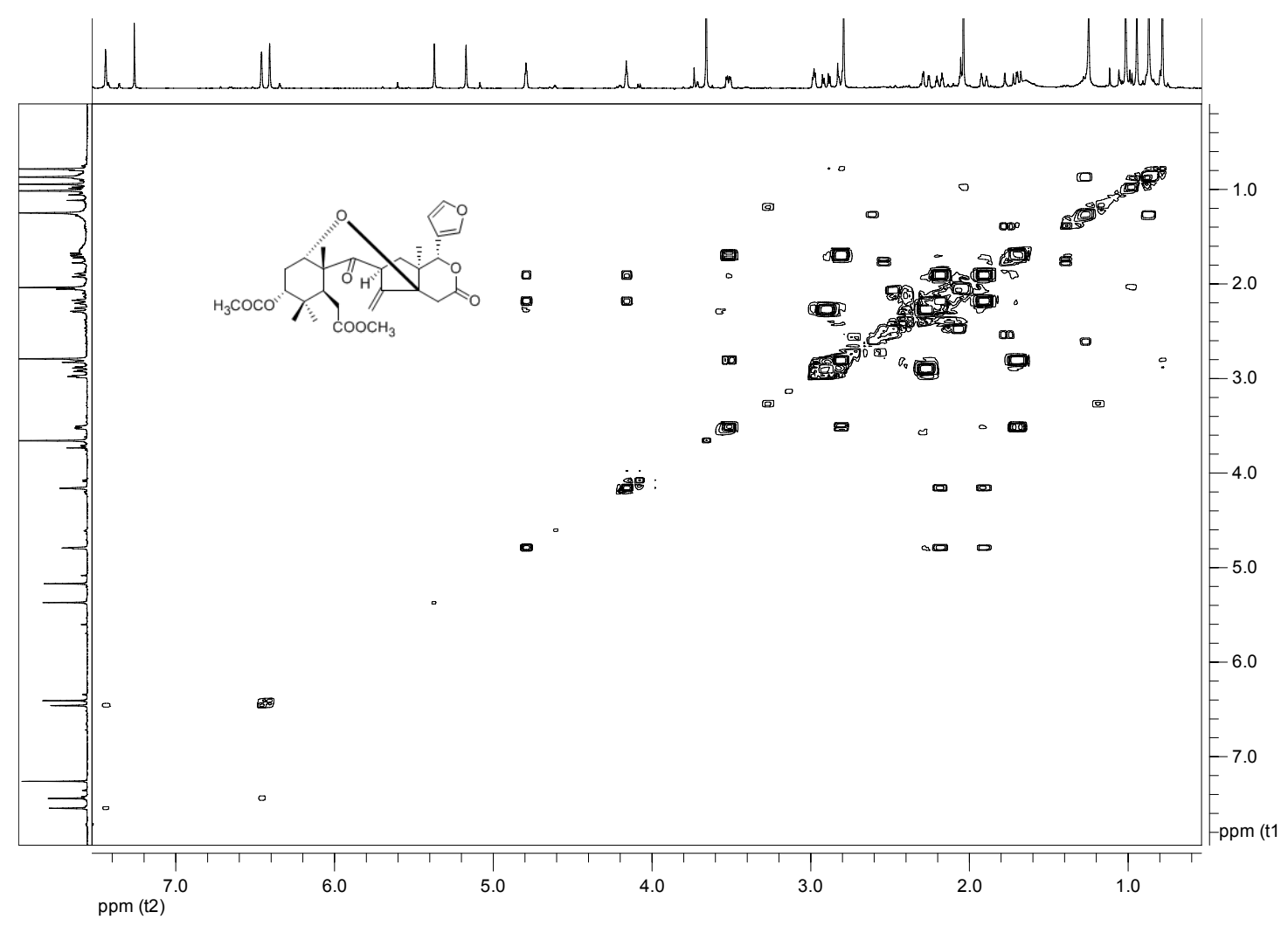

Figure S4. HMQC spectrum of Cipatrijugin A (1)

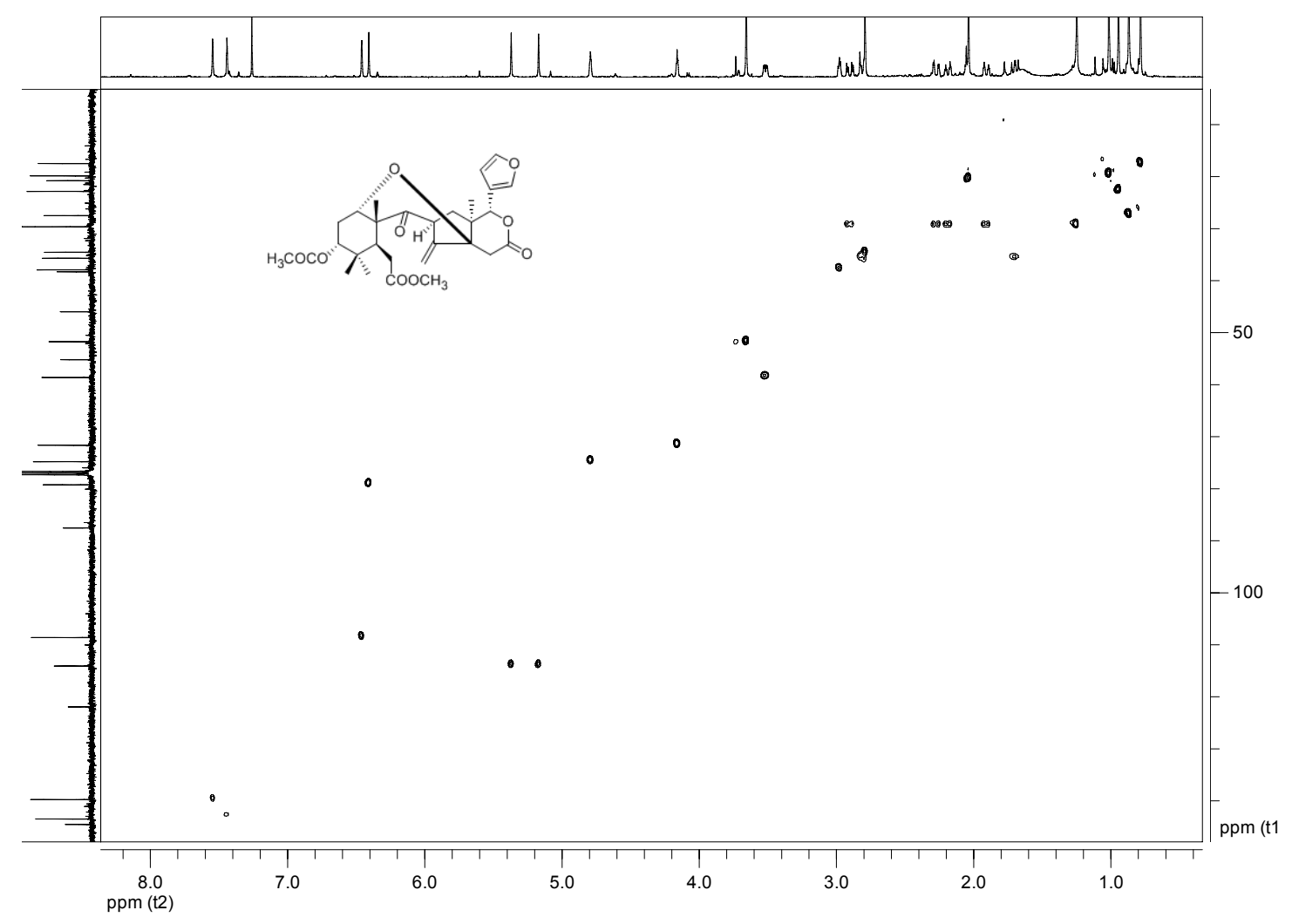


Figure S5. HMBC spectrum of Cipatrijugin A (1)

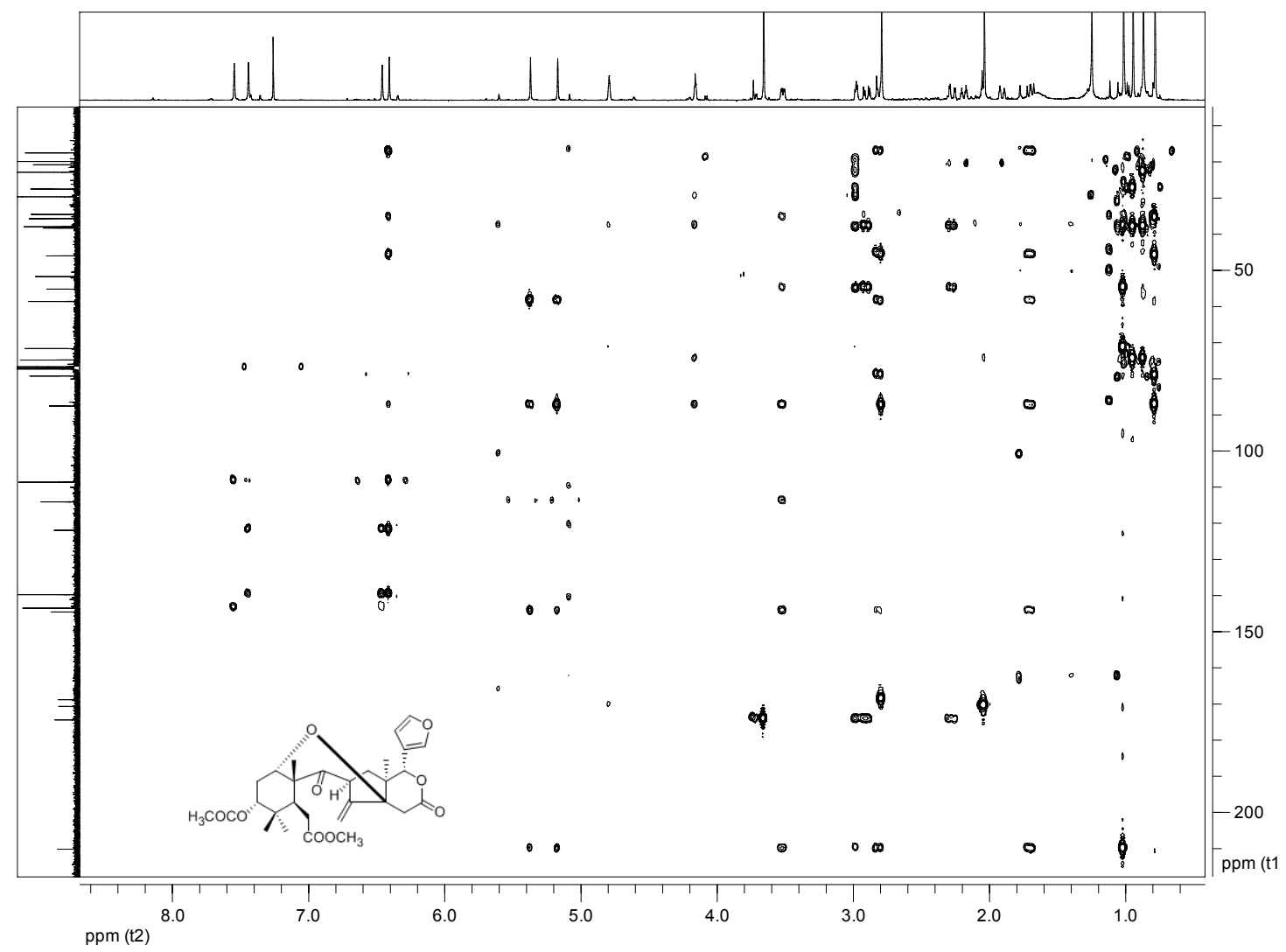

Figure S6. ROESY spectrum of Cipatrijugin A (1)

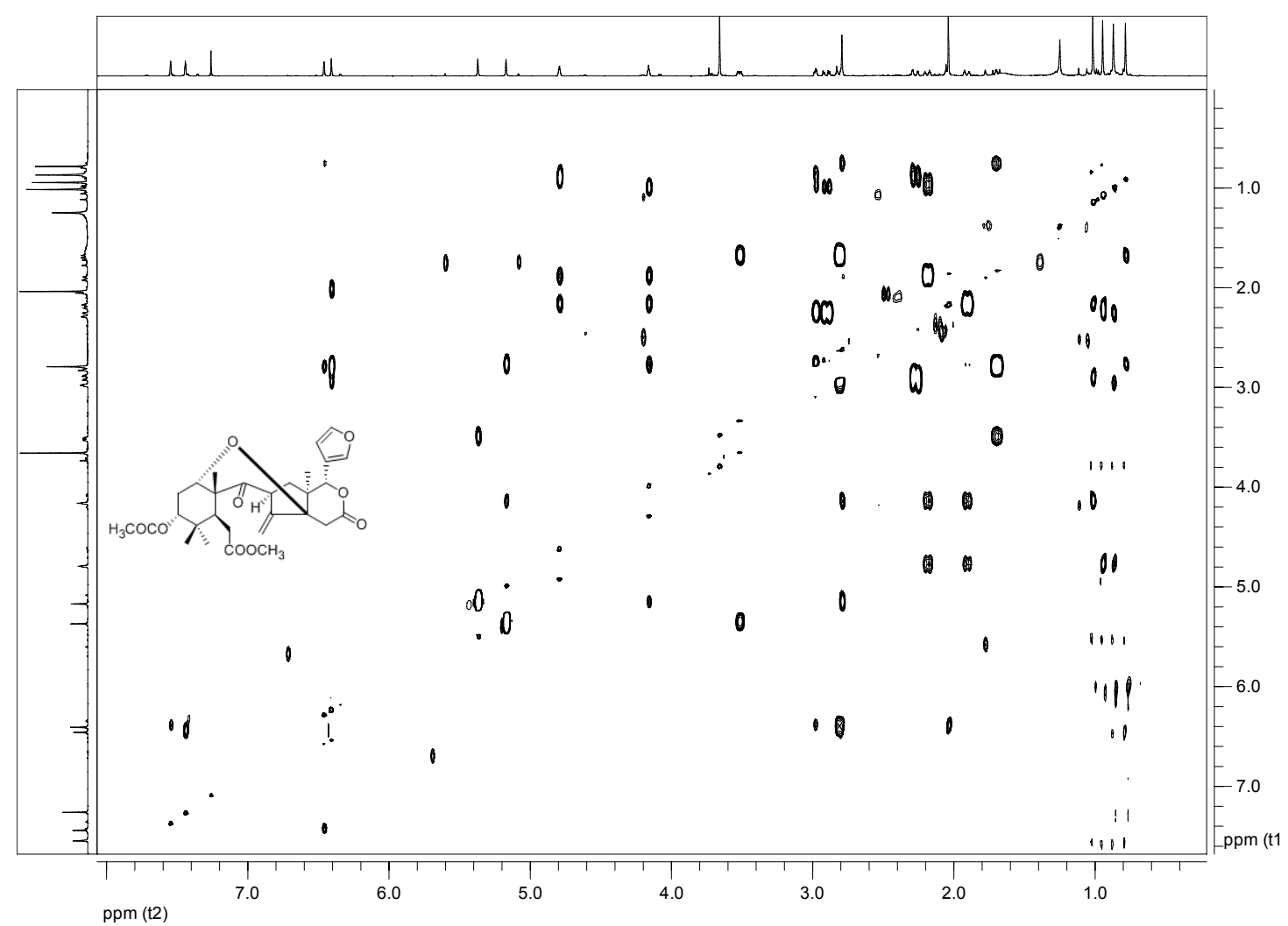


Figure S7. ${ }^{1} \mathrm{H}$ NMR spectrum of Cipatrijugin B (2)

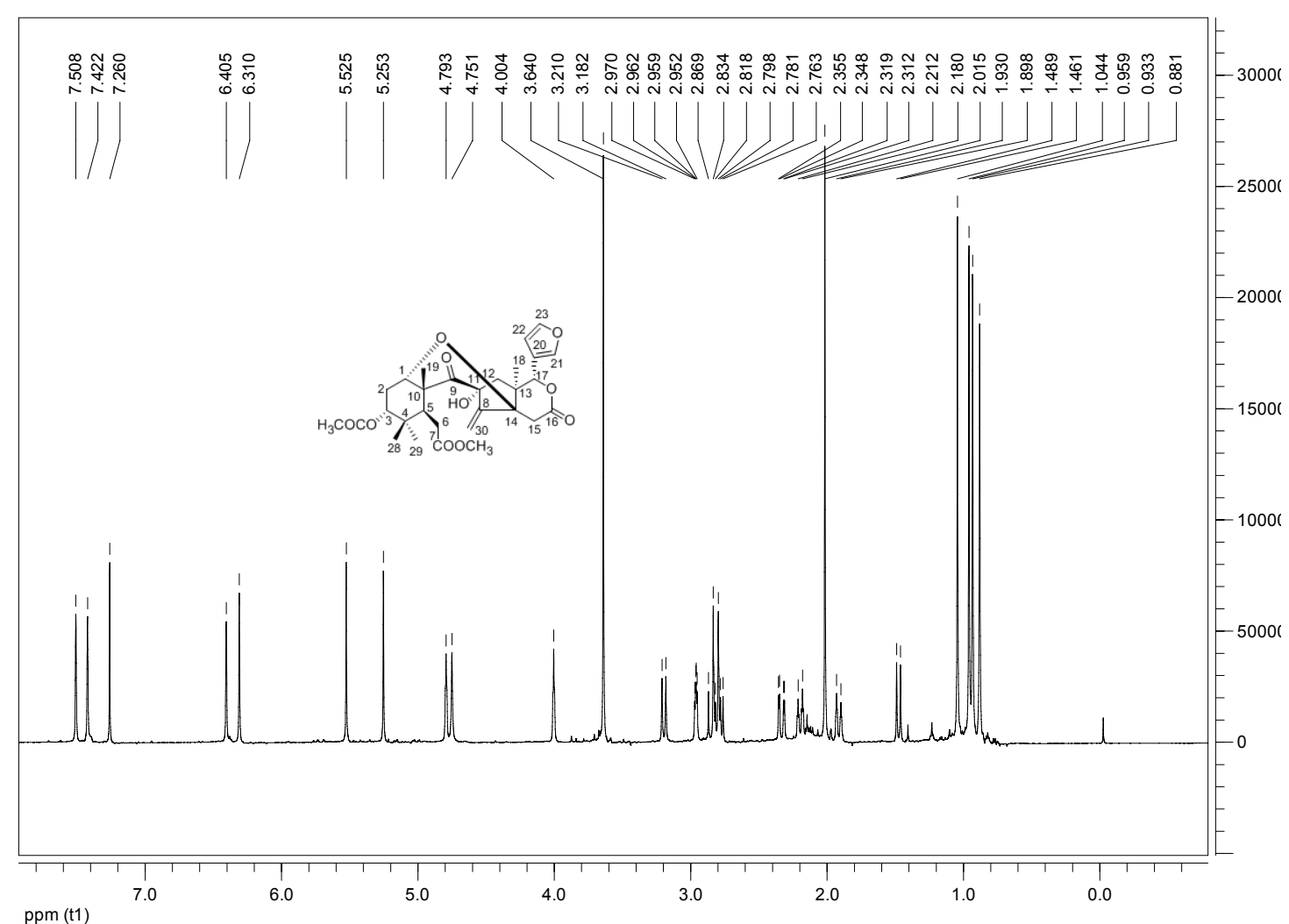

Figure S8. ${ }^{13}$ C NMR spectrum of Cipatrijugin B (2)

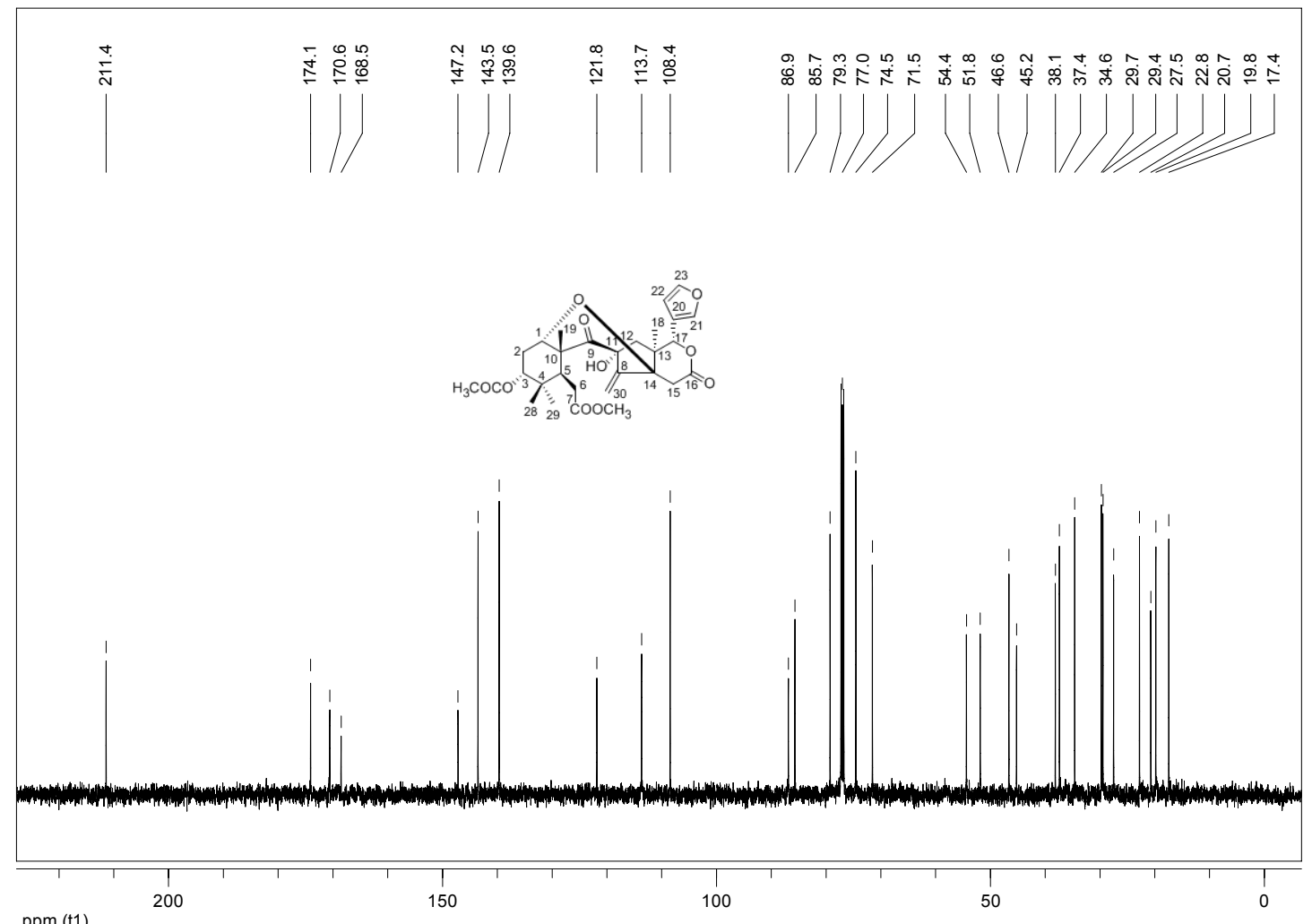

ppm (t1) 
Figure S9. HMQC spectrum of Cipatrijugin B (2)

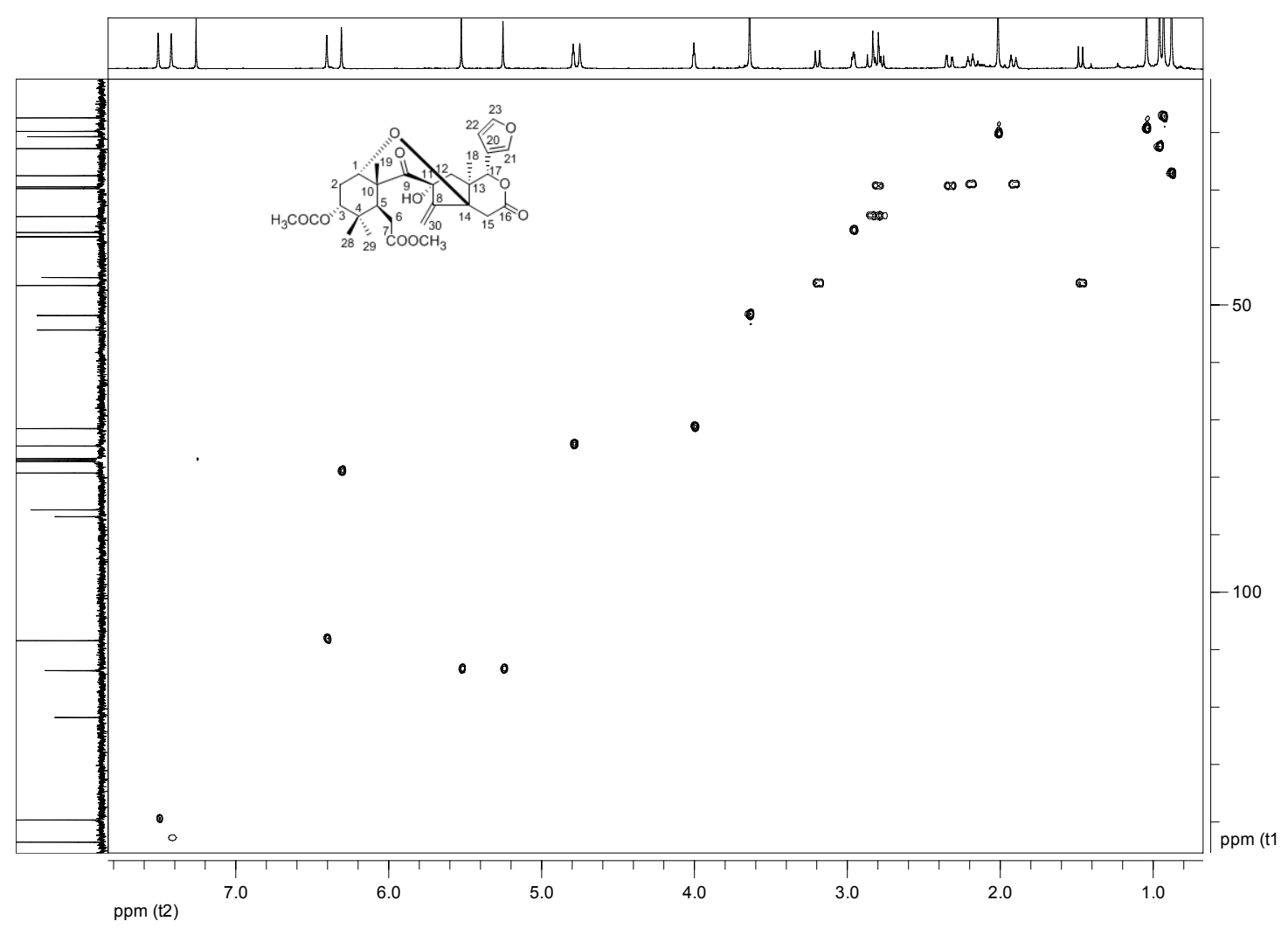

Figure S10. HMBC spectrumof Cipatrijugin B (2)

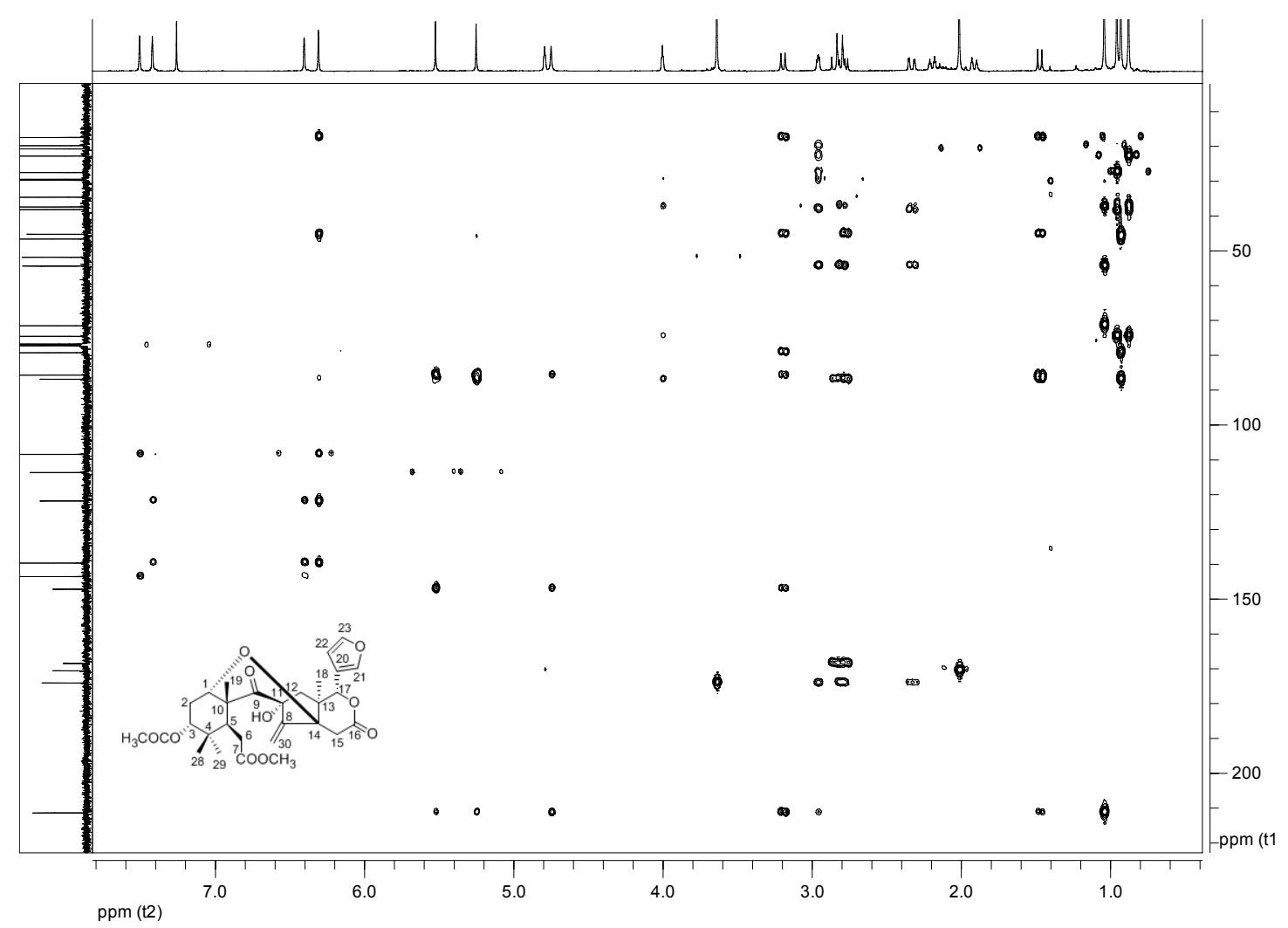


Figure S11. ${ }^{1} \mathrm{H}$ NMR spectrum of Cipatrijugin C (3)

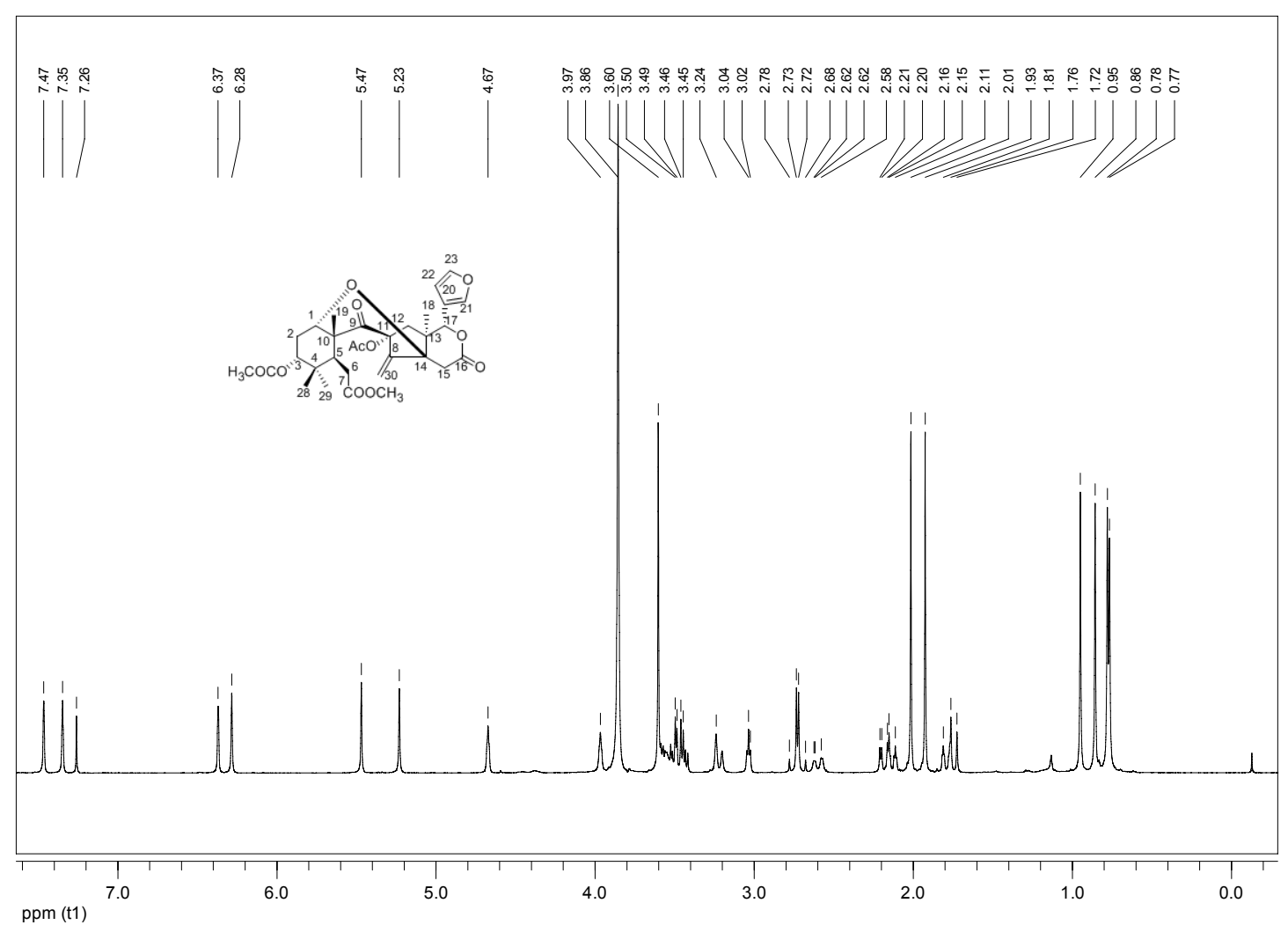

Figure S12. ${ }^{13} \mathrm{C}$ NMR spectrum of Cipatrijugin C (3)

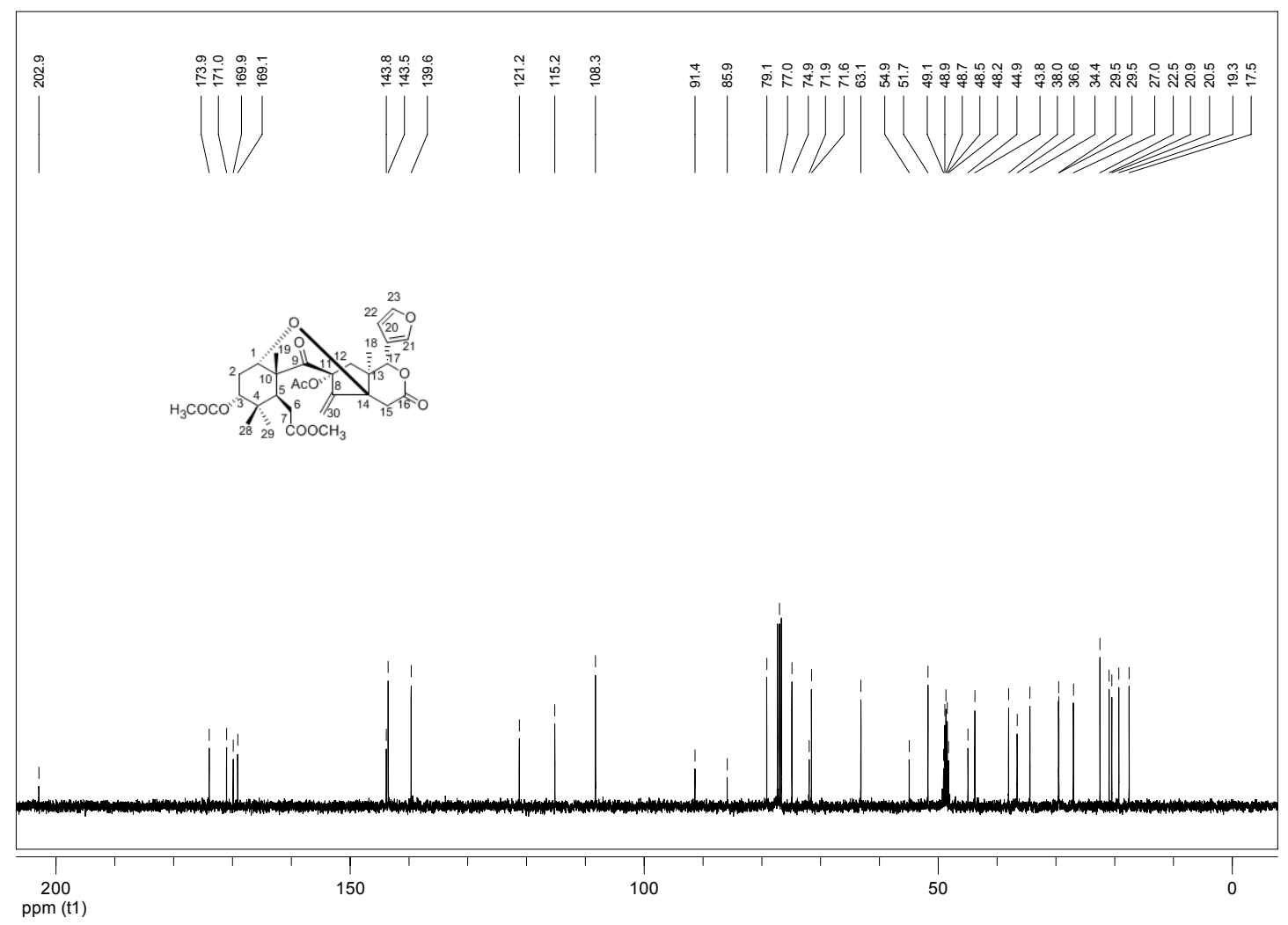


Figure S13. HMQC spectrum of Cipatrijugin C (3)

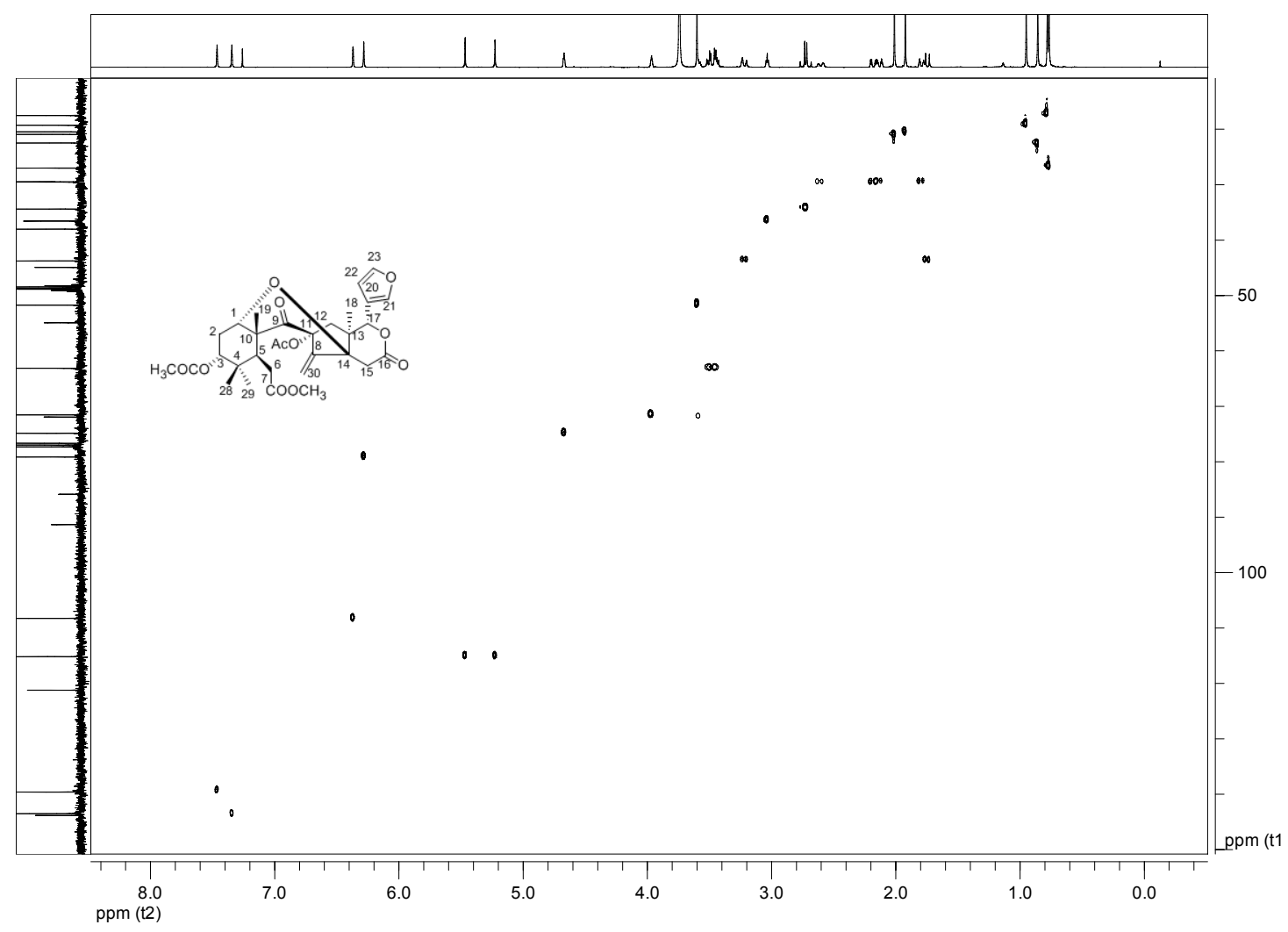

Figure S14. HMBC spectrum of Cipatrijugin C (3)

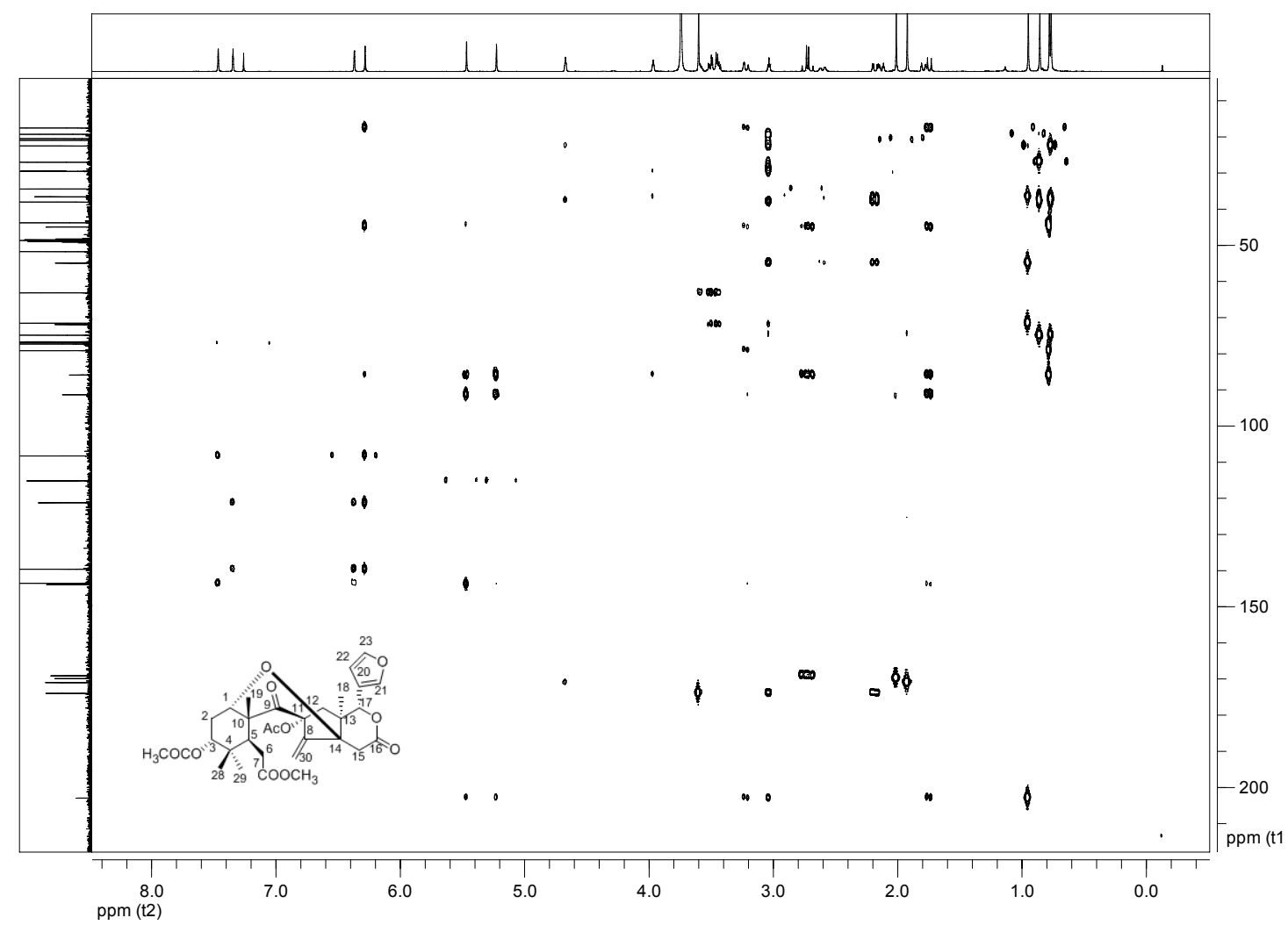


Figure S15. ${ }^{1} \mathrm{H}$ NMR spectrum of Cipatrijugin D (4)

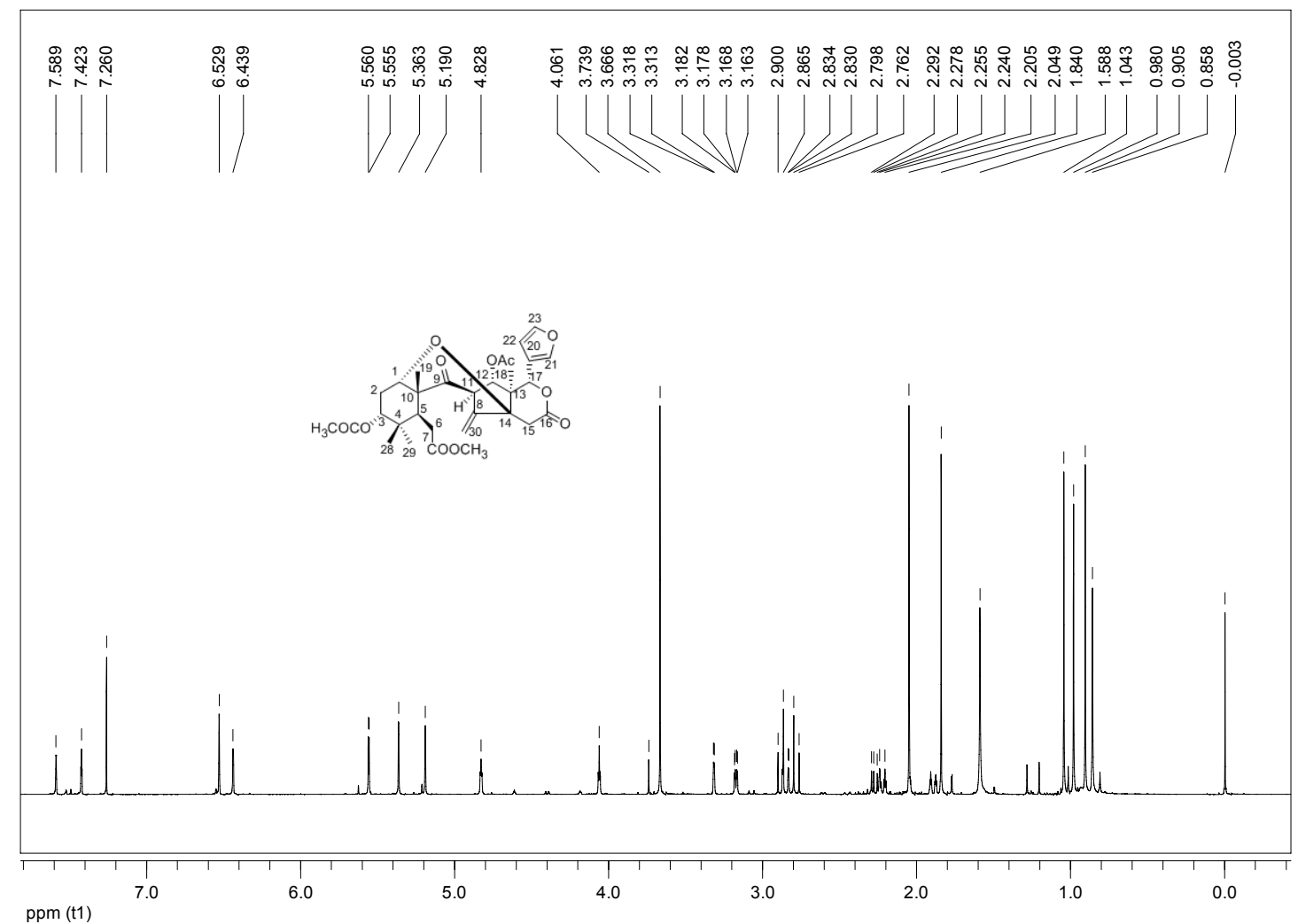

Figure S16. ${ }^{13} \mathrm{C}$ NMR spectrum of Cipatrijugin D (4)

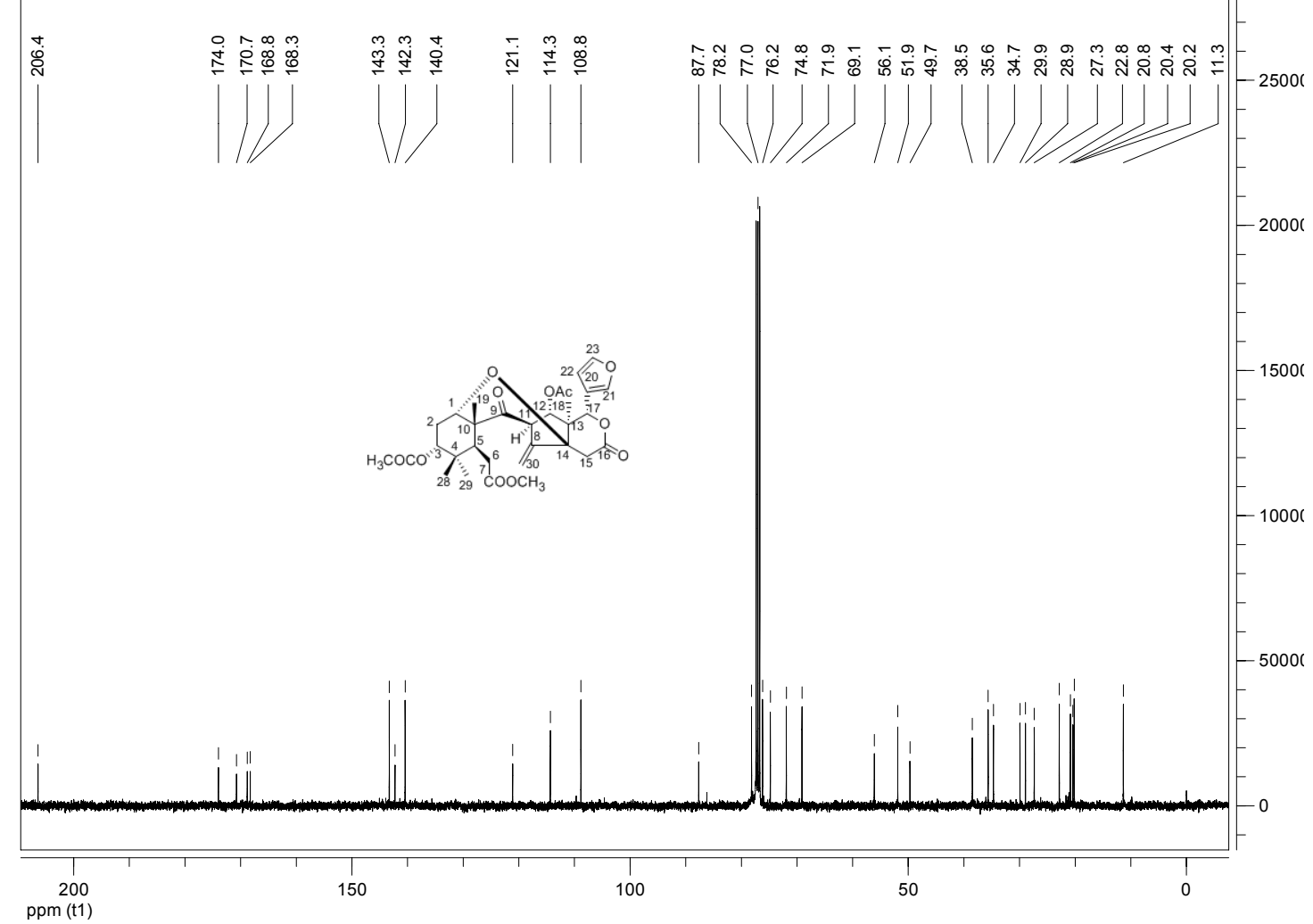


Figure S17. ${ }^{1} \mathrm{H}-{ }^{1} \mathrm{H}$ COSY spectrum of Cipatrijugin D (4)

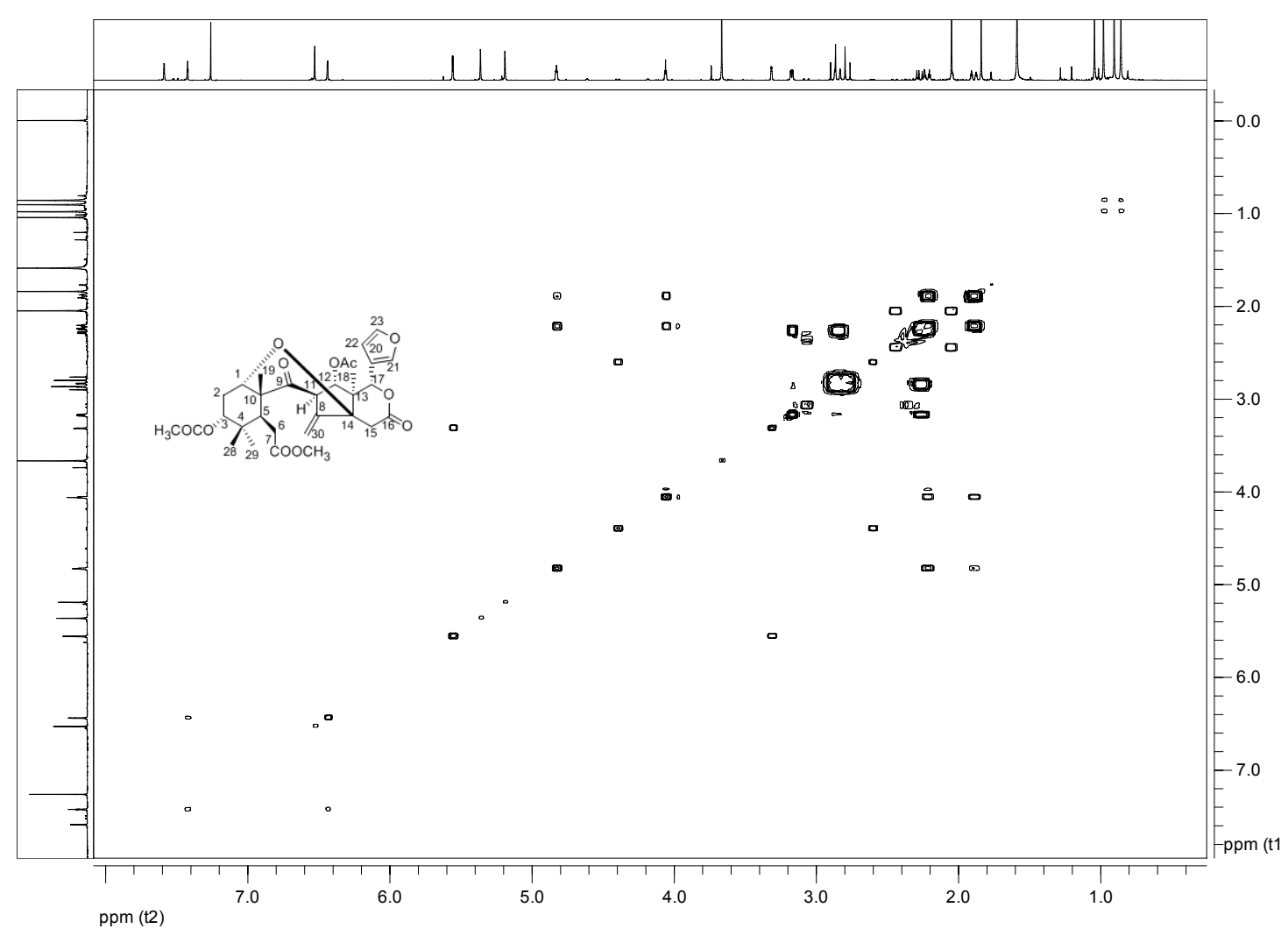

Figure S18. HMQC spectrum of Cipatrijugin D (4)

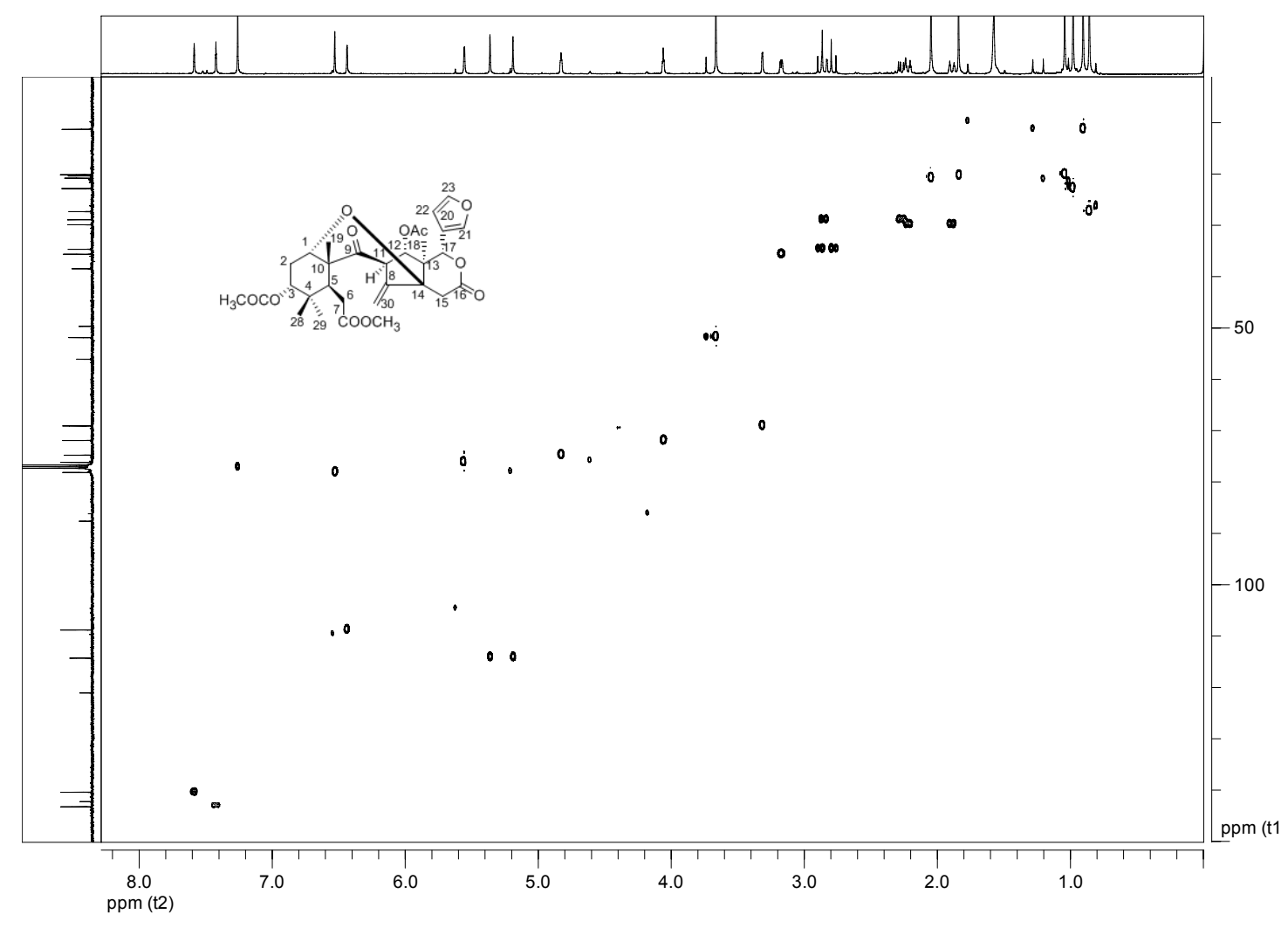


Figure S19. HMBC spectrum of Cipatrijugin D (4)

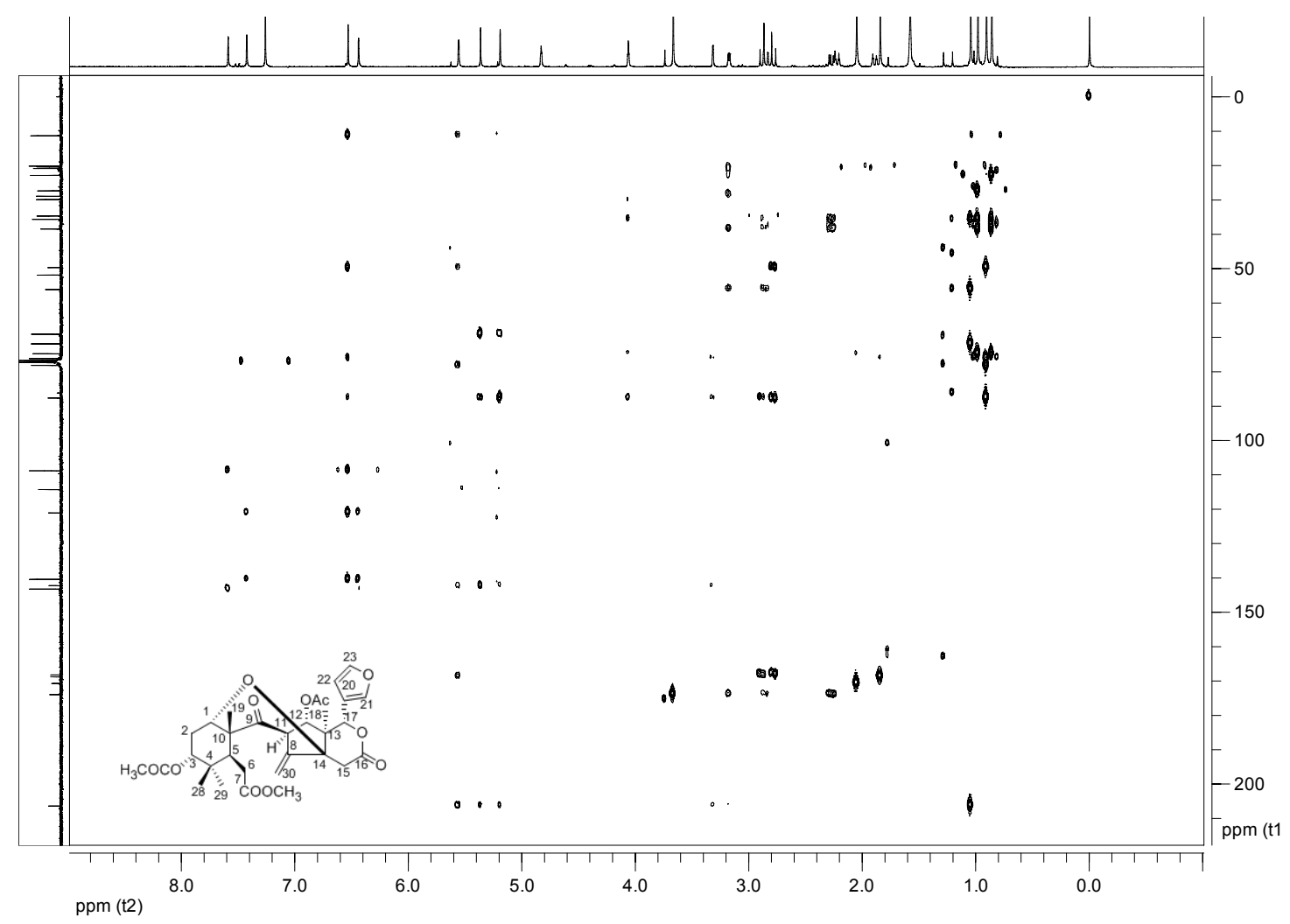

Figure S20. ROESY spectrum of Cipatrijugin D (4)

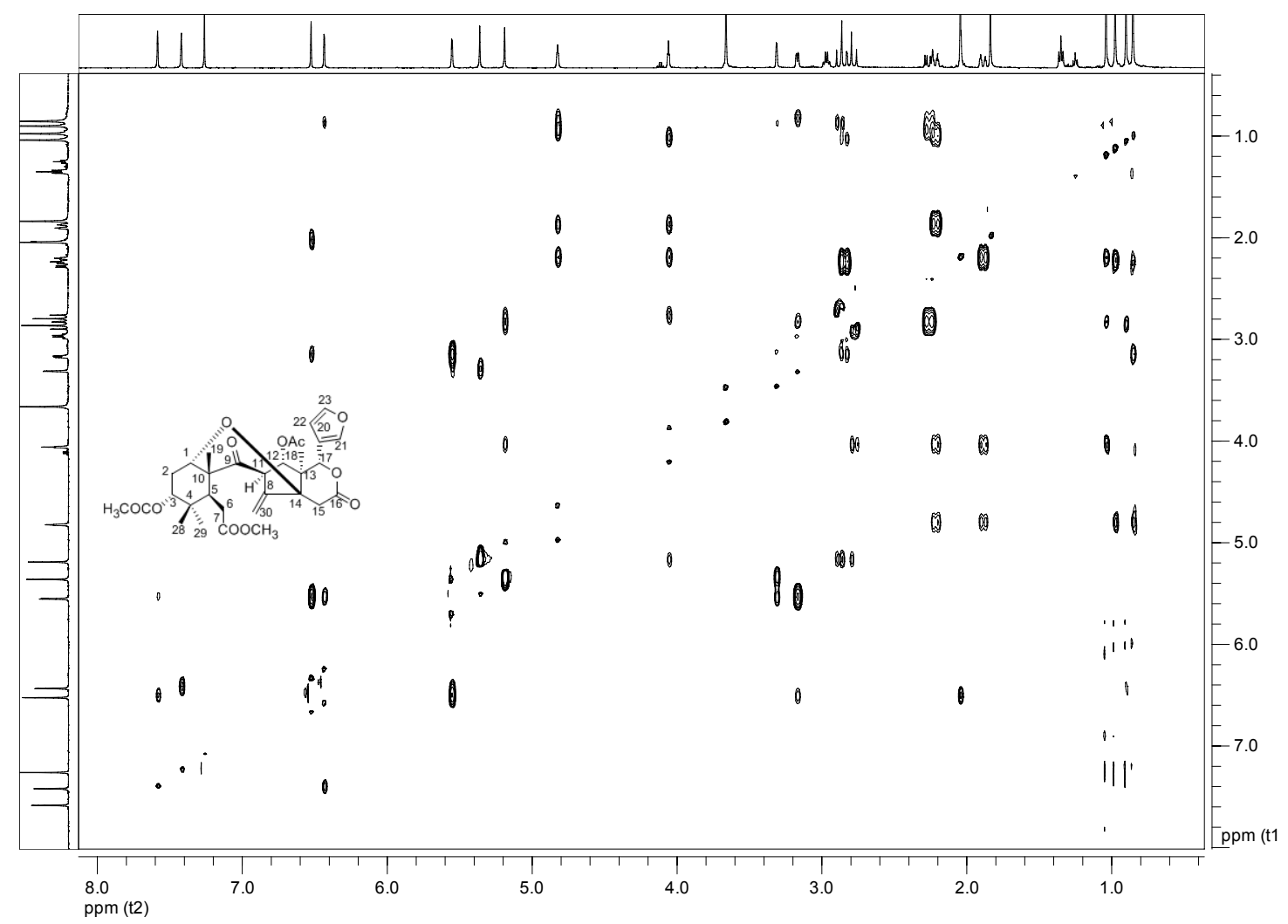


Structures of Cipatrijugins A (1) and D (4) were obtained at the B3LYP/3-21G(d) level of theory.

Cipatrijugin A (1)

Energy $=-1125689.15 \mathrm{Kcal} / \mathrm{mol}$

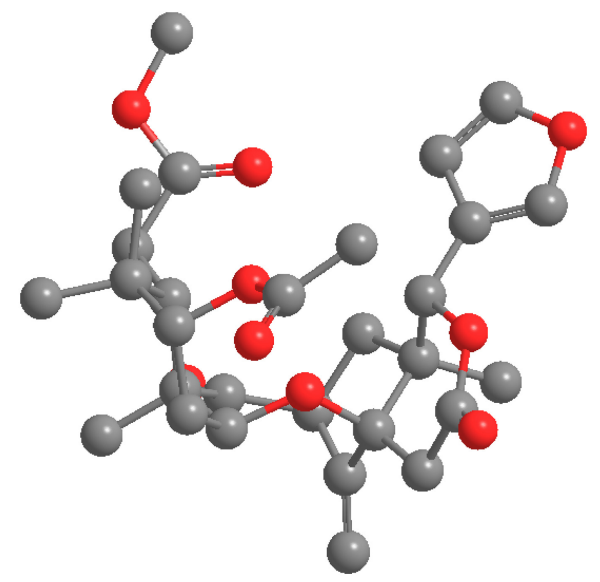

Figure S21. Standard Orientation of Cipatrijugin A (1)

\begin{tabular}{|c|c|c|c|c|c|}
\hline \multirow{2}{*}{$\begin{array}{l}\text { Center } \\
\text { Number }\end{array}$} & \multirow{2}{*}{$\begin{array}{l}\text { Atomic } \\
\text { Number }\end{array}$} & \multicolumn{4}{|c|}{ coordinates (Angstroms) } \\
\hline & & $\mathrm{X}$ & & & $\mathrm{Z}$ \\
\hline 1 & 6 & -4.3780 & 1.6370 & -3.2722 & \\
\hline 2 & 6 & -4.8672 & 0.1674 & -3.4112 & \\
\hline 3 & 6 & -3.8300 & -0.8173 & -2.9180 & \\
\hline 4 & 6 & -3.2935 & -0.4285 & -1.5366 & \\
\hline 5 & 8 & -3.3379 & 0.9144 & -1.1542 & \\
\hline 6 & 6 & -4.2909 & 1.9110 & -1.7604 & \\
\hline 7 & 6 & -5.5003 & 2.4341 & -4.0044 & \\
\hline 8 & 6 & -6.1794 & 1.3945 & -4.9937 & \\
\hline 9 & 6 & -5.3831 & 0.1108 & -4.8195 & \\
\hline 10 & 6 & -5.1594 & -0.8270 & -5.7367 & \\
\hline 11 & 6 & -7.6771 & 1.2462 & -4.6981 & \\
\hline 12 & 6 & -8.2574 & 0.4366 & -3.4988 & \\
\hline 13 & 8 & -8.4683 & 1.8375 & -5.4531 & \\
\hline 14 & 6 & -7.2505 & -0.5798 & -2.9020 & \\
\hline 15 & 6 & -7.7787 & -1.2433 & -1.6214 & \\
\hline 16 & 6 & -8.1716 & -0.2393 & -0.5235 & \\
\hline 17 & 6 & -9.1438 & 0.8621 & -1.0059 & \\
\hline 18 & 6 & -8.6115 & 1.4717 & -2.3539 & \\
\hline 19 & 6 & -3.0187 & 1.8536 & -3.9614 & \\
\hline 20 & 8 & -2.4020 & 4.9110 & -0.6720 & \\
\hline 21 & 6 & -2.5118 & 3.5419 & -0.9408 & \\
\hline
\end{tabular}


22

23

24

25

26

27

28

29

30

31

32

33

34

35

36

37

38

39

40

41

42

43

44

45

46

47

48

49

50

51

52

53

54

55

56

57

58

59

60

61

62

63

64

65
$-4.5138$

$-3.6556$

$-3.7675$

$-6.1355$

$-6.9684$

$-6.2507$

$-6.5334$

$-5.1485$

$-9.1763$

$-10.5681$

$-9.4750$

$-9.4926$

$-8.9230$

$-7.7283$

$-9.4547$

$-2.7588$

$-6.0247$

$-4.2376$

$-2.9767$

$-5.2649$

$-5.0692$

$-6.2279$

$-4.5556$

$-5.5639$

$-7.0219$

$-7.0100$

$-8.6530$

$-8.6175$

$-7.6387$

$-3.0485$

$-2.7845$

$-2.2188$

$-1.6514$

$-3.7476$

$-4.7340$

$-5.5440$

$-4.3585$

$-9.9143$

$-9.4531$

$-8.1929$

$-11.2735$

$-10.6029$

$-10.9161$
$-9.9156$

4.5001

$-1.3905$

5.4663

$-0.9642$

3.2545

$-1.3834$

1.7599

$-6.0218$

0.4491

0.0298

$-0.2835$

0.9774

$-1.4468$

1.2451

0.5614

1.5654

2.0007

0.0520

0.2498

$-1.0590$

$-0.3149$

$-4.0983$

2.6631

$-2.8204$

3.9570

$-2.2952$

4.2647

$-2.2427$

4.7982

$-1.8573$

6.1116

$-1.3497$

$-1.2287$

$-0.7878$

0.1117

$-2.4839$

$-1.8273$

$-2.8272$

$-0.8495 \quad-3.6052$

1.7190

$-1.3112$

3.2560

$-4.5811$

2.8624

$-3.3148$

$-1.7031$

$-5.5219$

$-0.7499$

$-6.7411$

$-1.3482$

$-3.6494$

$-1.9029$

$-1.2120$

$-1.8572$

$-1.8593$

$-0.7951$

0.3080

$\begin{array}{ll}1.8876 & -2.0830\end{array}$

$1.4892-4.9936$

2.9217

$-3.9682$

1.3409

$-3.4203$

2.9335

$-0.7399$

6.5250

$-0.8064$

0.0505

2.4346

1.5417

1.8441

0.7128

0.8213

2.7613

$-0.2207$

1.5859

1.0283

2.4641

0.1412

0.9321

$-1.5420$

$-0.7061$

$-1.5829$

1

$0.0800 \quad-0.0335$ 


$\begin{array}{llrrr}66 & 1 & -9.1403 & -0.9489 & -4.9285 \\ 67 & 1 & -9.9721 & -0.9487 & -3.3679 \\ 68 & 1 & -10.1901 & 0.4051 & -4.4995 \\ 69 & 1 & -10.5300 & 2.5791 & -2.5002 \\ 70 & 1 & -9.4791 & 2.7128 & -3.9158 \\ 71 & 1 & -10.3657 & 6.6204 & -1.0411 \\ 72 & 1 & -8.7730 & 5.9741 & -0.5075 \\ 73 & 1 & -8.9410 & 6.6656 & -2.1386 \\ 74 & 1 & -5.5519 & 4.6203 & -1.6594\end{array}$




\section{Cipatrijugin D (4)}

Energy $=-1267897.63 \mathrm{Kcal} / \mathrm{mol}$

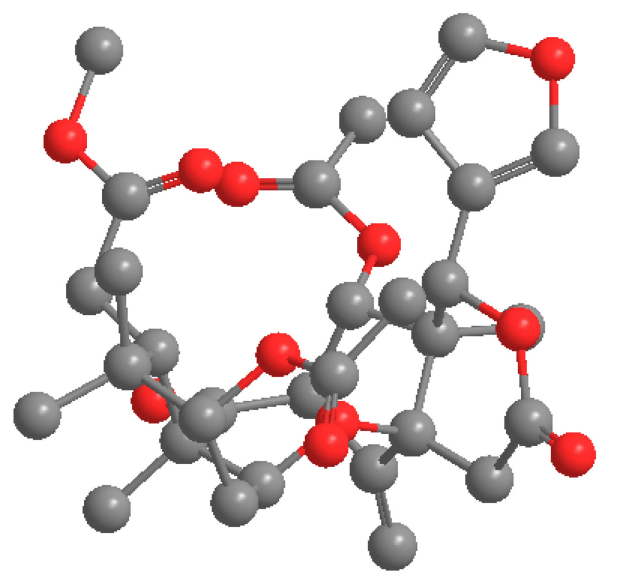

Figure S22. Standard Orientation of Cipatrijugin D (4)

\begin{tabular}{|c|c|c|c|c|}
\hline \multirow{2}{*}{$\begin{array}{l}\text { Center } \\
\text { Number }\end{array}$} & \multirow{2}{*}{$\begin{array}{l}\text { Atomic } \\
\text { Number }\end{array}$} & \multicolumn{3}{|c|}{ Coordinates (Angstroms) } \\
\hline & & $\mathrm{X}$ & $\mathrm{Y}$ & $\mathrm{Z}$ \\
\hline 1 & 6 & 4.7031 & 1.7836 & -1.9504 \\
\hline 2 & 6 & 4.3744 & 0.3364 & -2.4101 \\
\hline 3 & 6 & 4.9016 & 0.0578 & -3.7994 \\
\hline 4 & 6 & 6.3600 & 0.5021 & -3.9612 \\
\hline 5 & 8 & 6.8495 & 1.5154 & -3.1321 \\
\hline 6 & 6 & 6.2354 & 1.8510 & -1.8018 \\
\hline 7 & 6 & 3.9523 & 1.8461 & -0.5884 \\
\hline 8 & 6 & 2.7776 & 0.7962 & -0.6965 \\
\hline 9 & 6 & 2.9128 & 0.2028 & -2.0906 \\
\hline 10 & 6 & 1.9424 & -0.2887 & -2.8559 \\
\hline 11 & 6 & 2.8222 & -0.2622 & 0.4192 \\
\hline 12 & 6 & 3.8751 & -1.4100 & 0.4728 \\
\hline 13 & 8 & 1.9708 & -0.1856 & 1.3174 \\
\hline 14 & 6 & 4.5149 & -1.7136 & -0.9055 \\
\hline 15 & 6 & 5.6604 & -2.7312 & -0.8085 \\
\hline 16 & 6 & 6.7678 & -2.3215 & 0.1767 \\
\hline 17 & 6 & 6.2505 & -1.9408 & 1.5819 \\
\hline 18 & 6 & 5.0466 & -0.9424 & 1.4305 \\
\hline 19 & 6 & 4.1739 & 2.8290 & -2.9447 \\
\hline 20 & 6 & 7.3746 & 4.9704 & -0.1804 \\
\hline 21 & 1 & 6.4993 & 3.2310 & 0.8268 \\
\hline 22 & 6 & 6.8137 & 3.7349 & -0.0750 \\
\hline 23 & 6 & 7.3194 & 4.1172 & -2.2499 \\
\hline
\end{tabular}


24

25

26

27

28

29

30

31

32

33

34

35

36

37

38

39

40

41

42

43

44

45

46

47

48

49

50

51

52

53

54

55

56

57

58

59

60

61

62

63

64

65

66

67
7.7046

6.7711

1.8297

7.5262

8.4967

8.6374

9.2813

7.3912

5.9567

3.0765

4.5677

5.1392

5.8172

4.7855

5.0760

7.0863

5.1328

4.8643

4.3114

6.5564

3.4017

4.6010

2.1411

0.9103

3.7480

6.1168

5.2594

7.4876

5.4964

3.1272

4.2435

4.7742

7.6235

7.5285

10.1780

9.5414

8.6730

7.1407

8.3159

7.5609

5.4169

5.3762

6.9091

2.2506
5.2444

$3.1875-1.4197$

1.3192

$-1.1422$

$-1.4355$

$-2.5658$

$-0.1945$

$-1.2085$

$-3.2477$

$-2.6430$

$-0.3835$

1.0022

1.6618

1.4578

2.8891

0.0648

$-0.4882$

$-1.0076$

0.6021

1.0769

3.2075

1.6427

$-0.6773$

$-0.3137$

$-2.0844$

$-2.8614$

$-3.7011$

$-3.1434$

$-0.1039$

2.6294

3.8197

2.8102

5.7372

4.1256

$-0.4832$

0.3467

0.4674

$-1.1212$

$-1.7916$

$-0.2142$

$-3.0328$

$-3.9732$

$-3.7206$

$-2.8472$
$-0.5625$

$-0.3390$

$-1.2967$

$-1.7531$

$-1.6445$

2.3459

2.3639

0.9683

2.7871

3.0251

2.2301

4.2589

4.5177

$-4.8369$

$-1.4389$

$-4.0402$

$-4.5459$

$-1.1043$

$-0.4252$

0.2599

$-3.8498$

$-2.5209$

$-1.5946$

$-1.7928$

$-0.4978$

0.2493

0.8958

$-3.1915$

$-2.4989$

$-3.8575$

0.5300

$-3.3021$

$-2.1935$

$-0.7314$

$-2.2712$

3.4086

2.2659

1.9347

3.2917

1.7918

2.6308

0.2758 


\begin{tabular}{|c|c|c|c|c|}
\hline 68 & 1 & 3.6940 & -3.5352 & 1.0329 \\
\hline 69 & 1 & 2.6417 & -2.4321 & 1.9465 \\
\hline 70 & 1 & 4.8422 & -1.0171 & 3.6348 \\
\hline 71 & 1 & 3.4782 & -0.2752 & 2.8102 \\
\hline 72 & 1 & 4.7939 & 3.0434 & 5.5576 \\
\hline 73 & 1 & 6.1350 & 3.1012 & 4.3565 \\
\hline 74 & 1 & 4.4643 & 3.4834 & 3.8382 \\
\hline 75 & 6 & 3.1820 & 3.6648 & 0.8630 \\
\hline 76 & 8 & 3.2943 & 2.9556 & 1.8575 \\
\hline 77 & 6 & 2.8242 & 5.1307 & 0.8079 \\
\hline 78 & 1 & 2.5049 & 5.4699 & 1.7929 \\
\hline 79 & 1 & 3.7067 & 5.6937 & 0.4840 \\
\hline 80 & 1 & 2.0348 & 5.2924 & 0.0696 \\
\hline
\end{tabular}

